\title{
Krebs von den Lungen-6 Measurement
}

National Cancer Institute

\section{Source}

National Cancer Institute. Krebs von den Lungen-6 Measurement. NCI Thesaurus. Code C154724.

The determination of the amount of Krebs von den Lungen- 6 present in a sample. 